\title{
On conditional mean ergodic semigroups of random linear operators
}

\section{Xia Zhang*}

\section{"Correspondence:} xiazhangxz@gmail.com Department of Mathematics, School of Science, Tianjin Polytechnic University, Tianjin 300160, People's Republic of China

\begin{abstract}
In this article, we prove two forms of conditional mean ergodic theorem for a strongly continuous semigroup of random isometric linear operators generated by a semigroup of measure-preserving measurable isomorphisms, one of which generalizes and improves several known important results.
\end{abstract}

\section{Introduction and the main results}

The notion of a random normed module (briefly, an $R N$ module), which was first introduced in [1] and subsequently elaborated in [2], is a random generalization of that of a normed space. In the last 10 years, the theory of $R N$ modules together with their random conjugate spaces have undergone a systematic and deep development [3-9], in particular the random reflexivity based on the theory of random conjugate spaces and the study of semigroups of random linear operators have also obtained some substantial advances in $[6,8,10-12]$.

It is well known that the $(\varepsilon, \lambda)$-topology induced by the $L^{0}$-norm on an $R N$ module is exactly the topology of convergence in probability $P$. Actually, it is Mustari and Taylor that earlier observed the essence of the $(\varepsilon, \lambda)$-topology, studied probability theory in Banach spaces and did many excellent works $[13,14]$ under the framework of the special $R N$ module $L^{0}(\mathcal{E}, X)$, where $L^{0}(\mathcal{E}, X)$ is the $R N$ module of equivalence classes of $X$-valued random variables defined on a probability space $(\Omega, \mathcal{E}, P)$, see [4] or Section 2 for the construction of $L^{0}(\mathcal{E}, X)$. Motivated by these works, we have recently begun to study the mean ergodic theorem under the framework of $R N$ modules to obtain the mean ergodic theorem in the sense of convergence in probability, in particular we proved a mean ergodic theorem for a strongly continuous semigroup of random unitary operators defined on complete random inner product modules in [8] and further investigated the mean ergodicity for an almost surely bounded strongly continuous semigroup of random linear operators on a random reflexive $R N$ module in [11]. Based on these and motivated by the idea of $[15,16]$, the purpose of this article is to investigate the conditional mean ergodicity for a special semigroup of random linear operator on the $R N$ module $L_{\mathcal{F}}^{p}(\mathcal{E}, X)$ and the construction of $L_{\mathcal{F}}^{p}(\mathcal{E}, X)$ is detailed as follows.

Now let us first recall the construction of $L_{\mathcal{F}}^{p}(\mathcal{E})$ in [16]. Let $(\Omega, \mathcal{E}, P)$ be a probability space, $\mathcal{F}$ a sub $\sigma$-algebra of $\mathcal{E}$ and $\bar{L}^{0}(\mathcal{E})$ (or $L^{0}(\mathcal{E})$ ) the set of equivalence classes of $\mathcal{E}$-measurable extended real-valued (real-valued) random variables on $\Omega$. Let $\bar{L}_{+}^{0}(\mathcal{E})=\{\xi \in$ $\left.\bar{L}^{0}(\mathcal{E}) \mid \xi \geq 0\right\}$ and $L_{+}^{0}(\mathcal{E})=\left\{\xi \in L^{0}(\mathcal{E}) \mid \xi \geq 0\right\}$. Similarly, one can understand such notions as 
$\bar{L}^{0}(\mathcal{F}), L^{0}(\mathcal{F}), L_{+}^{0}(\mathcal{F})$ and $\bar{L}_{+}^{0}(\mathcal{F})$. Define the mapping $\|\cdot\|_{p}: \bar{L}^{0}(\mathcal{E}) \rightarrow \bar{L}_{+}^{0}(\mathcal{F})$ by

$$
\|x\|_{p}=\left[E\left(|x|^{p} \mid \mathcal{F}\right)\right]^{\frac{1}{p}}
$$

for any $x \in \bar{L}^{0}(\mathcal{E})$ and $1 \leq p<\infty$, where $E\left(|x|^{p} \mid \mathcal{F}\right)=\lim _{n \rightarrow \infty} E\left(|x|^{p} \wedge n \mid \mathcal{F}\right)$ denotes the extended conditional expectation, and let

$$
L_{\mathcal{F}}^{p}(\mathcal{E})=\left\{x \in L^{0}(\mathcal{E})\left|\|x \mid\|_{p} \in L_{+}^{0}(\mathcal{F})\right\},\right.
$$

then $\left(L_{\mathcal{F}}^{p}(\mathcal{E}),\|\cdot\|_{p}\right)$ is an $R N$ module. In fact, $L_{\mathcal{F}}^{p}(\mathcal{E})$ is exactly the $L^{0}(\mathcal{F})$-module generated by $L^{p}(\mathcal{E})$, namely $L_{\mathcal{F}}^{p}(\mathcal{E})=L^{0}(\mathcal{F}) \cdot L^{p}(\mathcal{E}):=\left\{\xi x \mid \xi \in L^{0}(\mathcal{F})\right.$ and $\left.x \in L^{p}(\mathcal{E})\right\}$, where $L^{p}(\mathcal{E})=\left\{x \in L^{0}(\mathcal{E}) \mid E\left[|x|^{p}\right]<\infty\right\}$. Further, motivated by the idea of constructing $L_{\mathcal{F}}^{p}(\mathcal{E})$, Guo in [3] constructed a more general $R N$ module $L_{\mathcal{F}}^{p}(S)$ and established the random conjugate representation theorem of this type of $R N$ module. Precisely speaking, let $(S,\|\cdot\|)$ be an $R N$ module over the scalar field $K$ with base $(\Omega, \mathcal{E}, P)$, the mapping $\|\cdot \cdot\|_{p}: S \rightarrow \bar{L}_{+}^{0}(\mathcal{F})$ is defined as follows for any $x \in S$ and $p \in[1, \infty)$ :

$$
\|x\|_{p}=\left[E\left(\|x\|^{p} \mid \mathcal{F}\right)\right]^{\frac{1}{p}}
$$

where $E\left(\|x\|^{p} \mid \mathcal{F}\right)=\lim _{n \rightarrow \infty} E\left(\|x\|^{p} \wedge n \mid \mathcal{F}\right)$ also denotes the extended conditional expectation and let

$$
L_{\mathcal{F}}^{p}(S)=\left\{x \in S \mid\|x\|_{p} \in L_{+}^{0}(\mathcal{F})\right\} .
$$

Then $\left(L_{\mathcal{F}}^{p}(S),\|\cdot\| \|_{p}\right)$ is an $R N$ module over $K$ with base $(\Omega, \mathcal{F}, P)$. If we take $S=L^{0}(\mathcal{E})$, then $L_{\mathcal{F}}^{p}(S)$ is exactly $L_{\mathcal{F}}^{p}(\mathcal{E})$; if we further take $S=L^{0}(\mathcal{E}, X)$, then $L_{\mathcal{F}}^{p}\left(L^{0}(\mathcal{E}, X)\right)$ (briefly, $L_{\mathcal{F}}^{p}(\mathcal{E}, X)$ ) is an $R N$ module over $K$ with base $(\Omega, \mathcal{F}, P)$.

We can now state the main results of this article as follows.

Theorem 1.1 Let $(\Omega, \mathcal{E}, P)$ be a probability space, $X$ a reflexive Banach space over $K$, $\left\{h_{t}: t \geq 0\right\}$ a semigroup of measure-preserving measurable isomorphisms on $(\Omega, \mathcal{E}, P), p$ an arbitrary positive number such that $1<p<\infty$ and $\mathcal{F}=\left\{A \in \mathcal{E} \mid h_{t}^{-1}(A)=A, \forall t \geq 0\right\}$. If $\lim _{t \rightarrow 0}\left\|x \circ h_{t}-x\right\|_{p}=0, \forall x \in L_{\mathcal{F}}^{p}(\mathcal{E}, X)$, then, for any $x \in L_{\mathcal{F}}^{p}(\mathcal{E}, X)$, there exists some $\bar{x} \in L_{\mathcal{F}}^{p}(\mathcal{E}, X)$ such that $\bar{x}=\bar{x} \circ h_{t}$ for each $t \geq 0$ and

$$
\lim _{r \rightarrow \infty} \frac{1}{r} \int_{0}^{r}\left(x \circ h_{t}\right) d t=\bar{x}
$$

in the $(\varepsilon, \lambda)$-topology induced by $\|\cdot \cdot\|_{p}$.

Theorem 1.2 Let $(\Omega, \mathcal{E}, P), X,\left\{h_{t}: t \geq 0\right\}$ and $\mathcal{F}$ be the same as in Theorem 1.1 and $\bar{p}$ a given positive number such that $1<\bar{p}<\infty$ and $\lim _{t \rightarrow 0}\left\|x \circ h_{t}-x\right\|_{\bar{p}}=0, \forall x \in L_{\mathcal{F}}^{\bar{p}}(\mathcal{E}, X)$. Then, for any $x \in L_{\mathcal{F}}^{1}(\mathcal{E}, X)$, there exists some $\bar{x} \in L_{\mathcal{F}}^{1}(\mathcal{E}, X)$ such that $\bar{x}=\bar{x} \circ h_{t}$ for each $t \geq 0$ and

$$
\lim _{r \rightarrow \infty} \frac{1}{r} \int_{0}^{r}\left(x \circ h_{t}\right) d t=\bar{x}
$$

in the $(\varepsilon, \lambda)$-topology induced by $\|\cdot \cdot\|_{1}$. In particular, if $x \in L^{1}(\Omega, \mathcal{E}, X)$, then $\bar{x}=E(x \mid \mathcal{F})$. 
The remainder of this article is organized as follows: in Section 2 we briefly recall some necessary basic notions and facts and Section 3 is devoted to the proof of our main results.

\section{Preliminaries}

In the sequel of this article, $(\Omega, \mathcal{E}, P)$ denotes a probability space, $K$ the scalar field $R$ of real numbers or $C$ of complex numbers, and $L^{0}(\mathcal{E}, K)$ the algebra of equivalence classes of $K$-valued $\mathcal{E}$-measurable random variables on $\Omega$ under the ordinary addition, scalar multiplication and multiplication operations on equivalence classes. Besides, let $L^{0}(\mathcal{E}), \bar{L}^{0}(\mathcal{E})$ and $L_{+}^{0}(\mathcal{E})$ be the same as in Section 1.

It is well known from [17] that $\bar{L}^{0}(\mathcal{E})$ is a complete lattice under the ordering $\leq: \xi \leq \eta$ if and only if $\xi^{0}(\omega) \leq \eta^{0}(\omega)$ for $P$-almost all $\omega$ in $\Omega$ (briefly, a.s.), where $\xi^{0}$ and $\eta^{0}$ are arbitrarily chosen representatives of $\xi$ and $\eta$, respectively. Furthermore, every subset $A$ of $\bar{L}^{0}(\mathcal{E})$ has a supremum, denoted by $\bigvee A$, and an infimum, denoted by $\bigwedge A$, and there exist two sequences $\left\{a_{n}, n \in N\right\}$ and $\left\{b_{n}, n \in N\right\}$ in $A$ such that $\bigvee_{n \geq 1} a_{n}=\vee A$ and $\bigwedge_{n \geq 1} b_{n}=\bigwedge A$. Finally, $L^{0}(\mathcal{E})$, as a sublattice of $\bar{L}^{0}(\mathcal{E})$, is complete in the sense that every subset with an upper bound has a supremum.

Definition $2.1[2,3]$ An ordered pair $(S,\|\cdot\|)$ is called an $R N$ module over $K$ with base $(\Omega, \mathcal{E}, P)$ if $S$ is a left module over the algebra $L^{0}(\mathcal{E}, K)$ and $\|\cdot\|$ is a mapping from $S$ to $L_{+}^{0}(\mathcal{E})$ such that the following three axioms are satisfied:

(1) $\|\xi x\|=|\xi|\|x\|, \forall \xi \in L^{0}(\mathcal{E}, K)$ and $x \in S$;

(2) $\|x+y\| \leq\|x\|+\|y\|, \forall x, y \in S$;

(3) $\|x\|=0$ implies $x=\theta$ (the null vector of $S$ ), where $\|\cdot\|$ is called the $L^{0}$-norm on $S$ and $\|x\|$ is called the $L^{0}$-norm of a vector $x \in S$.

It should be pointed out that the following idea of introducing the $(\varepsilon, \lambda)$-topology is due to Schweizer and Sklar [18].

Let $(S,\|\cdot\|)$ be an $R N$ module over $K$ with base $(\Omega, \mathcal{E}, P)$. For any positive real numbers $\varepsilon$ and $\lambda$ such that $\lambda<1$, let $N_{\theta}(\varepsilon, \lambda)=\{x \in S \mid P\{\omega \in \Omega \mid\|x\|(\omega)<\varepsilon\}>\lambda\}$, then $\left\{N_{\theta}(\varepsilon, \lambda) \mid \varepsilon>\right.$ $0,0<\lambda<1\}$ is a local base at the null vector $\theta$ of some Hausdorff linear topology. The linear topology is called the $(\varepsilon, \lambda)$-topology. In this article, given an $R N$ module $(S,\|\cdot\|)$ over $K$ with base $(\Omega, \mathcal{E}, P)$, it is always assumed that $(S,\|\cdot\|)$ is endowed with the $(\varepsilon, \lambda)$ topology. One only needs to notice that a sequence $\left\{x_{n}, n \in N\right\}$ in $S$ converges to $x \in S$ in the $(\varepsilon, \lambda)$-topology if and only if $\left\{\left\|x_{n}-x\right\|, n \in N\right\}$ converges to 0 in probability $P$.

Example Let $X$ be a normed space over $K$ and $L^{0}(\mathcal{E}, X)$ the linear space of equivalence classes of $X$-valued $\mathcal{E}$-random variables on $\Omega$. The module multiplication operation $\cdot: L^{0}(\mathcal{E}, K) \times L^{0}(\mathcal{E}, X) \rightarrow L^{0}(\mathcal{E}, X)$ is defined by $\xi x=$ the equivalence class of $\xi^{0} x^{0}$, where $\xi^{0}$ and $x^{0}$ are the respective arbitrarily chosen representatives of $\xi \in L^{0}(\mathcal{E}, K)$ and $x \in L^{0}(\mathcal{E}, X)$, and $\left(\xi^{0} x^{0}\right)(\omega)=\xi^{0}(\omega) \cdot x^{0}(\omega), \forall \omega \in \Omega$. Furthermore, the mapping $\|\cdot\|:$ $L^{0}(\mathcal{E}, X) \rightarrow L_{+}^{0}(\mathcal{E})$ by $\|x\|=$ the equivalence class of $\left\|x^{0}\right\|, \forall x \in L^{0}(\mathcal{E}, X)$, where $x^{0}$ is as above. Then it is easy to see that $\left(L^{0}(\mathcal{E}, X),\|\cdot\|\right)$ is an $R N$ module over $K$ with base $(\Omega, \mathcal{E}, P)$.

Definition $2.2[19]$ Let $\left(S^{1},\|\cdot\|_{1}\right)$ and $\left(S^{2},\|\cdot\|_{2}\right)$ be two $R N$ modules over $K$ with base $(\Omega, \mathcal{E}, P)$. A linear operator $T$ from $S^{1}$ to $S^{2}$ is called a random linear operator, further, the random linear operator $T$ is called a.s. bounded if there exists some $\xi \in L_{+}^{0}(\mathcal{E})$ such that $\|T x\|_{2} \leq \xi \cdot\|x\|_{1}$ for any $x \in S^{1}$. Denote by $B\left(S^{1}, S^{2}\right)$ the linear space of a.s. bounded 
random linear operators from $S^{1}$ to $S^{2}$, define $\|\cdot\|: B\left(S^{1}, S^{2}\right) \rightarrow L_{+}^{0}(\mathcal{E})$ by $\|T\|:=\bigwedge\{\xi \in$ $\left.L_{+}^{0}(\mathcal{E}) \mid\|T x\|_{2} \leq \xi \cdot\|x\|_{1}, \forall x \in S^{1}\right\}$ for any $T \in B\left(S^{1}, S^{2}\right)$, then it is easy to see that $\left(B\left(S^{1}, S^{2}\right)\right.$, $\|\cdot\|)$ is an $R N$ module over $K$ with base $(\Omega, \mathcal{E}, P)$.

Specially, denote $\left(B\left(S^{1}, S^{2}\right),\|\cdot\|\right)$ by $\left(S^{*},\|\cdot\|^{*}\right)$ when $\left(S^{1},\|\cdot\|_{1}\right)$ is a given $R N$ module $(S,\|\cdot\|)$ over $K$ with base $(\Omega, \mathcal{E}, P)$ and $S^{2}=L^{0}(\mathcal{E}, K)$, then $\left(S^{*},\|\cdot\|^{*}\right)$ is called the random conjugate space of $(S,\|\cdot\|)$. Let $\left(S^{* *},\|\cdot\|^{* *}\right)$ be the random conjugate space of $\left(S^{*},\|\cdot\|^{*}\right)$. The canonical embedding mapping $J: S \rightarrow S^{* * *}$ defined by $(J x)(f)=f(x)$ for any $x \in S$ and $f \in S^{\prime \prime}$, is random-norm preserving. If $J$ is surjective, then $S$ is called random reflexive [10].

Definition $2.3[8,11]$ Let $(S,\|\cdot\|)$ be an $R N$ module over $K$ with base $(\Omega, \mathcal{E}, P), B(S)$ the set of a.s. bounded random linear operators on $S$. A family $\{B(t): t \geq 0\} \subset B(S)$ is called a semigroup of random linear operators if

$$
B(0)=I \quad \text { and } \quad B(s) B(t)=B(s+t)
$$

for all $s, t \geq 0$, where $I$ denotes the identity operator on $S$. Further, if the mapping $B(\cdot) x$ : $[0,+\infty) \rightarrow S, t \mapsto B(t) x$ is continuous w.r.t. the $(\varepsilon, \lambda)$-topology for every $x \in S$, then the semigroup of random linear operators $\{B(t): t \geq 0\}$ is said to be strongly continuous. Besides, if $\bigvee_{t \geq 0}\|B(t)\| \in L_{+}^{0}(\mathcal{E})$, then $\{B(t): t \geq 0\}$ is called an a.s. bounded strongly continuous semigroup of random linear operators.

Proposition $2.4[19]$ Let $\left(S^{1},\|\cdot\|_{1}\right)$ and $\left(S^{2},\|\cdot\|_{2}\right)$ be two $R N$ modules over $K$ with base $(\Omega, \mathcal{E}, P)$. Then we have the following statements:

(1) $T \in B\left(S^{1}, S^{2}\right)$ if and only if $T$ is a continuous module homomorphism;

(2) If $T \in B\left(S^{1}, S^{2}\right)$, then $\|T\|=\vee\left\{\|T x\|_{2}: x \in S^{1}\right.$ and $\left.\|x\|_{1} \leq 1\right\}$, where 1 denotes the identity element in $L^{0}(\mathcal{E})$.

\section{Proof of the main results}

The proof of Theorem 1.2 needs Theorem 1.1 and Lemma 3.5 below. To prove Theorem 1.1 and introduce Lemma 3.5, we will first recall the definition of Riemann integral for abstract-valued functions from a finite real interval to an $R N$ module and a sufficient condition for such a function to be Riemann-integrable.

Let $[a, b]$ be a finite real interval and $\mathcal{P}=\left\{x_{1}, x_{2}, \ldots, x_{k}, \ldots, x_{n}\right\}$ a finite partition into $[a, b]$, namely, $a=x_{1}<x_{2}<\cdots<x_{n-1}<x_{n}=b$ and $\lambda(\mathcal{P})=\max _{1 \leq i \leq n}\left\{\Delta x_{i}\right\}$, where $\Delta x_{i}=x_{i}-x_{i-1}(i=$ $1, \ldots, n)$. Besides, in the following of this section we always suppose that $(S,\|\cdot\|)$ denotes a complete $R N$ module over $K$ with base $(\Omega, \mathcal{E}, P)$.

Definition 3.1 [8] Let $f$ be a function from $[a, b]$ to $S . f$ is called Riemann-integrable on $[a, b]$ if there exists some $I$ in $S$ with the following property: for any positive numbers $\varepsilon$ and $\lambda$ with $\lambda<1$ there is a positive number $\delta(\varepsilon, \lambda)$ such that

$$
P\left\{\omega \in \Omega \mid\left\|\sum_{i=1}^{n} f\left(\xi_{i}\right) \Delta x_{i}-I\right\|(\omega)<\varepsilon\right\}>1-\lambda
$$

for any finite partition $\mathcal{P}=\left\{x_{1}, x_{2}, \ldots, x_{k}, \ldots, x_{n}\right\}$ and arbitrarily chosen $\xi_{i} \in\left[x_{i-1}, x_{i}\right](1 \leq$ $i \leq n)$ whenever $\lambda(\mathcal{P})<\delta(\varepsilon, \lambda)$. Further $I$ is called the Riemann integral of $f$ in the $(\varepsilon, \lambda)$ topology over $[a, b]$, denoted by $\int_{a}^{b} f(t) d t$. 
Proposition 3.2 [8] Let $f$ be a continuous function from $[a, b]$ to $S$ such that $\bigvee_{t \in[a, b]}\|f(t)\| \in L_{+}^{0}(\mathcal{E})$, then $f$ is Riemann integrable in the $(\varepsilon, \lambda)$-topology on $[a, b]$. Further, if $\bigvee_{t \in[a, b]}\|f(t)\| \in L^{2}(\Omega, \mathcal{E}, R)$, then $f$ is Riemann integrable in $\|\cdot\|_{2}$ on $[a, b]$, where $\|\cdot\|_{2}$ denotes the 2-norm of the Banach space $L^{2}(\Omega, \mathcal{E}, R)$.

Definition 3.3 [11] Let $\{B(t): t \geq 0\}$ be an a.s. bounded strongly continuous semigroup of random linear operators on an $R N$ module $S$. We denote by

$$
C(r) x:=\frac{1}{r} \int_{0}^{r} B(s) x d s, \quad \forall x \in S, r>0
$$

the Cesàro means of $\{B(t): t \geq 0\}$. For any $x \in S$, if $\{C(r) x, r>0\}$ converges to some point in $S$ as $r \rightarrow \infty$, then $\{B(t): t \geq 0\}$ is called mean ergodic.

Note that it is Proposition 3.2 that makes Definition 3.3 be well defined.

Proposition $3.4[11]$ Let $(S,\|\cdot\|)$ be a complete $R N$ module over $K$ with base $(\Omega, \mathcal{E}, P)$. If $S$ is random reflexive, then every a.s. bounded strongly continuous semigroup of random linear operators on $S$ is mean ergodic.

It is known from [9] that $L^{0}(\mathcal{E}, X)$ is random reflexive if and only if $X$ is a reflexive Banach space. Besides, Guo [3] proved that if an $R N$ module $S$ is random reflexive, then $L_{\mathcal{F}}^{p}(S)$ is also random reflexive. Based on these facts as well as the preceding Proposition 3.4, we can now prove Theorem 1.1 as follows.

Proof of Theorem 1.1 For each $t \geq 0$, define the mapping $T(t): L_{\mathcal{F}}^{p}(\mathcal{E}, X) \rightarrow L_{\mathcal{F}}^{p}(\mathcal{E}, X)$ by $T(t) x=x \circ h_{t}$ for each $x \in L_{\mathcal{F}}^{p}(\mathcal{E}, X)$, then it is clear that $T(t)$ is a module homomorphism. Furthermore,

$$
T(0) x=x \circ h_{0}=x
$$

for any $x \in L_{\mathcal{F}}^{p}(\mathcal{E}, X)$ and

$$
T(s) T(t) x=\left(x \circ h_{t}\right) \circ h_{s}=x \circ h_{t+s}=T(s+t) x
$$

for any $s, t \geq 0$ and $x \in L_{\mathcal{F}}^{p}(\mathcal{E}, X)$, i.e. $\{T(t): t \geq 0\}$ is a semigroup of random linear operators on $L_{\mathcal{F}}^{p}(\mathcal{E}, X)$. 
Since $E\left(\|T(t) x\|^{p} \wedge n \mid \mathcal{F}\right), E\left(\|x\|^{p} \wedge n \mid \mathcal{F}\right) \in L^{1}(\Omega, \mathcal{F}, P)$ for each $t \geq 0, x \in L_{\mathcal{F}}^{p}(\mathcal{E}, X)$ and $n \in N$, it follows that

$$
\begin{gathered}
\int_{A} E\left(\|T(t) x\|^{p} \wedge n \mid \mathcal{F}\right)(\omega) P(d \omega) \\
=\int_{A}\left(\left[\|T(t) x\|^{p}(\omega)\right] \wedge n\right) P(d \omega) \\
=\int_{A}\left(\left\|x\left(h_{t}(\omega)\right)\right\|^{p} \wedge n\right) P(d \omega) \\
=\int_{h_{t}^{-1}(A)}\left(\left\|x\left(h_{t}(\omega)\right)\right\|^{p} \wedge n\right) P(d \omega) \\
=\int_{A}\left(\|x(\omega)\|^{p} \wedge n\right) P(d \omega) \\
=\int_{A} E\left(\|x\|^{p} \wedge n \mid \mathcal{F}\right)(\omega) P(d \omega)
\end{gathered}
$$

for any $A \in \mathcal{F}$. Thus we have

$$
E\left(\|T(t) x\|^{p} \wedge n \mid \mathcal{F}\right)=E\left(\|x\|^{p} \wedge n \mid \mathcal{F}\right)
$$

for any $t \geq 0, x \in L_{\mathcal{F}}^{p}(\mathcal{E}, X)$ and $n \in N$, letting $n \rightarrow \infty$ in (4) yields that

$$
E\left(\|T(t) x\|^{p} \mid \mathcal{F}\right)=E\left(\|x\|^{p} \mid \mathcal{F}\right)
$$

namely $\|T(t) x\|_{p}^{p}=\|x\|_{p}^{p}$ for any $x \in L_{\mathcal{F}}^{p}(\mathcal{E}, X)$, which shows that

$$
T(t):\left(L_{\mathcal{F}}^{p}(\mathcal{E}, X),\|\| \cdot \|_{p}\right) \rightarrow\left(L_{\mathcal{F}}^{p}(\mathcal{E}, X),\|\| \cdot \|_{p}\right)
$$

is a random isometric linear operator for each $t \geq 0$. Moreover,

$$
\lim _{t \rightarrow 0}\left\|x \circ h_{t}-x\right\|_{p}=0, \quad \forall x \in L_{\mathcal{F}}^{p}(\mathcal{E}, X) .
$$

Thus $\{T(t): t \geq 0\}$ is a strongly continuous semigroup of random isometric linear operators on $L_{\mathcal{F}}^{p}(\mathcal{E}, X)$.

Clearly, $\{T(t): t \geq 0\}$ is an a.s. bounded strongly continuous semigroup of random linear operators on $L_{\mathcal{F}}^{p}(\mathcal{E}, X)$. Since $X$ is reflexive, it follows that the $R N$ module $L^{0}(\mathcal{E}, X)$ is random reflexive, further, $L_{\mathcal{F}}^{p}(\mathcal{E}, X)$ is random reflexive. Thus it follows from Proposition 3.4 that $T(t)$ is mean ergodic for each $t \geq 0$, i.e. for any $x \in L_{\mathcal{F}}^{p}(\mathcal{E}, X)$ there exists some $\bar{x} \in L_{\mathcal{F}}^{p}(\mathcal{E}, X)$ such that

$$
\lim _{r \rightarrow \infty} \frac{1}{r} \int_{0}^{r}\left(x \circ h_{t}\right) d t=\lim _{r \rightarrow \infty} \frac{1}{r} \int_{0}^{r} T(t) x d t=\bar{x} .
$$

Now it remains to show that $\bar{x}=\bar{x} \circ h_{t}$ for each $t \geq 0$. In fact, since

$$
T(s)\left(\frac{1}{r} \int_{0}^{r} T(t) x d t\right)=\frac{1}{r} \int_{0}^{r} T(s+t) x d t=\frac{1}{r} \int_{s}^{r+s} T(t) x d t
$$


and

$$
\begin{aligned}
\frac{1}{r} & \int_{s}^{r+s} T(t) x d t \\
& =\frac{1}{r} \int_{0}^{r+s} T(t) x d t-\frac{1}{r} \int_{0}^{s} T(t) x d t \\
& =\frac{r+s}{r} \frac{1}{r+s} \int_{0}^{r+s} T(t) x d t-\frac{1}{r} \int_{0}^{s} T(t) x d t,
\end{aligned}
$$

for any $r, s>0$ and $x \in S$, fix $s$ and $x$, letting $r \rightarrow \infty$ in (5) yields

$$
\lim _{r \rightarrow \infty} T(s)\left(\frac{1}{r} \int_{s}^{r+s} T(t) x d t\right)=\bar{x}
$$

Further, $T(t)$ is a random isometric linear operator for each $t \geq 0$, thus we have $T(t) \bar{x}=\bar{x}$, namely $\bar{x}=\bar{x} \circ h_{t}$.

This completes the proof.

It should be pointed out that Lemma 3.5 is merely mentioned in [8] and partially proved in [12].

Lemma 3.5 Let $f$ be a continuous function from $[a, b]$ to $L^{0}(\mathcal{E})$ such that $\bigvee_{t \in[a, b]}|f(t)| \in$ $L_{+}^{0}(\mathcal{E})$. Then

$$
\int_{\Omega}\left[\int_{a}^{b} f(t) d t\right] d P=\int_{a}^{b}\left[\int_{\Omega} f(t) d P\right] d t
$$

Proof Let $\xi=\bigvee_{t \in[a, b]}|f(t)|$, then $\xi \in L_{+}^{0}(\mathcal{E})$. Let $H_{m}=[m-1 \leq \xi<m]$ for each $m \in N$, then $\left\{H_{m}, m \in N\right\}$ is a sequence of pairwise disjoint $\mathcal{F}$-measurable sets such that $\sum_{m=1}^{\infty} H_{m}=$ $\Omega$. Define a mapping $f_{m}:[a, b] \rightarrow S$ by $f_{m}(t)=I_{H_{m}} \cdot f(t)$ for each $m \in N$. Obviously, $\bigvee_{t \in[a, b]}\left|f_{m}(t)\right| \in L^{2}(\Omega, \mathcal{E}, R)$ for each $m \in N$ and each $t \in[a, b]$, thus it follows by Proposition 3.2 that $f_{m}$ is Riemann integrable in $\|\cdot\|_{2}$ on $[a, b]$. Let $\mathcal{P}, \lambda(\mathcal{P})$ and $\Delta x_{i}, \xi_{i}(1 \leq i \leq n)$ be the same as in Definition 3.1 and $I_{m}=\int_{a}^{b} f_{m}(t) d t, I_{m n}=\sum_{i=1}^{n} f_{m}\left(\xi_{i}\right) \Delta x_{i}$ for each $m \in N$. Since

$$
\begin{aligned}
& \left|\int_{\Omega} I_{m n} d P-\int_{\Omega} I_{m} d P\right| \\
& \quad \leq \int_{\Omega}\left|I_{m n}-I_{m}\right| d P \\
& \quad \leq\left[\int_{\Omega}\left|I_{m n}-I_{m}\right|^{2} d P\right]^{\frac{1}{2}} \\
& \quad=\left\|I_{m n}-I_{m}\right\|_{2}
\end{aligned}
$$

for each fixed $m \in N$, we have

$$
\int_{\Omega} I_{m} d P=\lim _{\lambda(\mathcal{P}) \rightarrow 0} \int_{\Omega} I_{m n} d P=\lim _{\lambda(\mathcal{P}) \rightarrow 0} \sum_{i=1}^{n} \int_{\Omega} f_{m}\left(\xi_{i}\right) d P \Delta x_{i}=\int_{a}^{b}\left[\int_{\Omega} f_{m}(t) d P\right] d t .
$$


Thus for any $k \in N$, we have

$$
\sum_{m=1}^{k} \int_{\Omega}\left[\int_{a}^{b} f_{m}(t) d t\right] d P=\sum_{m=1}^{k} \int_{a}^{b}\left[\int_{\Omega} f_{m}(t) d P\right] d t
$$

Since

$$
\sum_{m=1}^{\infty} P\left(H_{m}\right)=P\left(\sum_{m=1}^{\infty} H_{m}\right)=P(\Omega)
$$

it follows that $\left\{\sum_{m=1}^{k} \int_{\Omega}\left[\int_{a}^{b} f_{m}(t) d t\right] d P, k \in N\right\}$ and $\left\{\sum_{m=1}^{k} \int_{a}^{b}\left[\int_{\Omega} f_{m}(t) d P\right] d t, k \in N\right\}$ are both Cauchy sequences. Letting $k \rightarrow \infty$ in Equation (9) yields Equation (7).

We can now prove Theorem 1.2.

Proof of Theorem 1.2 Let $T(t) x=x \circ h_{t}$ for each $t \geq 0$ and $x \in L_{\mathcal{F}}^{\bar{p}}(\mathcal{E}, X)$, then it follows from Theorem 1.1 that

$$
\frac{1}{r} \int_{0}^{r}\left(x \circ h_{t}\right) d t \rightarrow \bar{x} \quad(n \rightarrow \infty)
$$

in the $(\varepsilon, \lambda)$-topology induced by $\||\cdot|\|_{\bar{p}}$. Thus by the random Schwarz-Cauchy inequality, we have

$$
\left\|\frac{1}{r} \int_{0}^{r}\left(x \circ h_{t}\right) d t-\bar{x}\right\|_{1} \rightarrow 0 \quad(n \rightarrow \infty)
$$

in probability $P$. For any $x \in L_{\mathcal{F}}^{1}(\mathcal{E}, X)$, since

$$
\left\|\frac{1}{r} \int_{0}^{r}\left(x \circ h_{t}\right) d t\right\|\left\|_{1}=\right\| \frac{1}{r} \int_{0}^{r} T(t) x d t\|\|_{1} \leq\|x\|_{1}
$$

for each $n \in N$ and $L_{\mathcal{F}}^{\bar{p}}(\mathcal{E}, X)$ is dense in $L_{\mathcal{F}}^{1}(\mathcal{E}, X)$ in $\|\cdot\| \|_{1}$, one can obtain the desired Equation (2).

If $x \in L^{1}(\Omega, \mathcal{E}, X)$, it suffices to show that $\bar{x}=E(x \mid \mathcal{F})$. Let $A \in \mathcal{F}$, then for each $n \in N$, it follows from Lemma 3.5 that

$$
\begin{aligned}
\int_{A}\left[\frac{1}{r} \int_{0}^{r} T(t) x d t\right] d P & =\frac{1}{r} \int_{0}^{r}\left[\int_{A} T(t) x d P\right] d t \\
& =\frac{1}{r} \int_{0}^{r}\left[\int_{A} x\left(h_{t}(\omega)\right) P(d \omega)\right] d t \\
& =\frac{1}{r} \int_{0}^{r}\left[\int_{h_{t}^{-1}(A)} x\left(h_{t}(\omega)\right) P(d \omega)\right] d t \quad\left(\text { since } h_{t}^{-1}(A)=A\right) \\
& =\frac{1}{r} \int_{0}^{r}\left[\int_{A} x(\omega) P(d \omega)\right] d t \\
& =\int_{A} x(\omega) P(d \omega) .
\end{aligned}
$$


Furthermore, since

$$
\lim _{n \rightarrow \infty} \frac{1}{r} \int_{0}^{r} T(t) x d t=\bar{x}
$$

in the $(\varepsilon, \lambda)$-topology induced by $\|\cdot \cdot\|_{1}$ and

$$
\left\|\frac{1}{r} \int_{0}^{r}\left(x \circ h_{t}\right) d t\right\|_{1} \leq\|x\|_{1}
$$

for each $x \in L^{1}(\Omega, \mathcal{E}, X) \subset L_{\mathcal{F}}^{1}(\mathcal{E}, X)$, letting $r \rightarrow \infty$ in (10), it follows from the Lebesgue's dominated convergence theorem that

$$
\int_{A} \bar{x}(\omega) P(d \omega)=\int_{A} x(\omega) P(d \omega)
$$

namely the desired result follows.

Let $H$ be a Hilbert space over $K$. It is clear that Theorem 1.2 still holds when $X$ is taken place of $H$, which includes the following known result.

Corollary 3.6 [12] Let $(\Omega, \mathcal{E}, P)$ be a probability space, $\left\{h_{t}: t \geq 0\right\}$ a semigroup of measurepreserving measurable isomorphisms on $(\Omega, \mathcal{E}, P), \mathcal{F}=\left\{A \in \mathcal{E} \mid h_{t}^{-1}(A)=A, \forall t \geq 0\right\}$ and $\lim _{t \rightarrow 0}\left\|x \circ h_{t}-x\right\|_{2}=0, \forall x \in L_{\mathcal{F}}^{2}(\mathcal{E}, H)$. Then, for any $x \in L_{\mathcal{F}}^{1}(\mathcal{E}, H)$, there exists some $\bar{x} \in L_{\mathcal{F}}^{1}(\mathcal{E}, H)$ such that $\bar{x}=\bar{x} \circ h_{t}$ for each $t \geq 0$ and

$$
\lim _{r \rightarrow \infty} \frac{1}{r} \int_{0}^{r}\left(x \circ h_{t}\right) d t=\bar{x}
$$

Corollary 3.7 If, in addition to the hypothesis of Theorem 1.2, we assume that $A \in \mathcal{F}$ if and only if $P(A)=0$ of 1 , then $\bar{x}=E(x)$.

\section{Competing interests}

The author declare that they have no competing interests.

\section{Acknowledgement}

The author would like to express his sincere gratitude to Prof. Guo Tiexin for his invaluable suggestions. The study was supported by the National Natural Science Foundation of China (No. 11171015). The author would also like to thank the referees for their invaluable suggestions.

Received: 7 February 2012 Accepted: 8 May 2012 Published: 3 July 2012

\section{References}

1. Guo, TX: Extension theorems of continuous random linear operators on random domains. J. Math. Anal. Appl. 193, $15-27(1995)$

2. Guo, TX: Some basic theories of random normed linear spaces and random inner product spaces. Acta Anal. Funct. Appl. 1, 160-184 (1999)

3. Guo, TX: Relations between some basic results derived from two kinds of topologies for a random locally convex module. J. Funct. Anal. 258, 3024-3047 (2010)

4. Guo, TX: Recent progress in random metric theory and its applications to conditional risk measures. Sci. China Ser. A 54(4), 633-660 (2011)

5. Guo, TX, Zeng, XL: Random strict convexity and random uniform convexity in random normed modules. Nonlinear Anal. 73, 1239-1263 (2010)

6. Guo, TX, Li, SB: The James theorem in complete random normed modules. J. Math. Anal. Appl. 308, $257-265$ (2005)

7. Guo, TX, Shi, G: The algebraic structure of finitely generated $L^{0}(\mathcal{F}, K)$-modules and the Helly theorem in random normed modules. J. Math. Anal. Appl. 381, 833-842 (2011) 
8. Guo, TX, Zhang, X: Stone's representation theorem of a group of random unitary operators on complete complex random inner product modules. Sci. Sin. Math. 42(3), 181-202 (2012) (in Chinese)

9. Guo, TX: The Radon-Nikodým property of conjugate spaces and the $w^{*}$-equivalence theorem for $w^{*}$-measurable functions. Sci. China Ser. A 39, 1034-1041 (1996)

10. Guo, TX: A characterization for a complete random normed module to be random reflexive. J. Xiamen Univ., Nat. Sci. 36, 499-502 (1997) (in Chinese)

11. Zhang, X: On mean ergodic semigroups of random linear operators. Proc. Jpn. Acad., Ser. A, Math. Sci. 88, 53-58 (2012)

12. Zhang, X, Guo, TX: Von Neumann's mean ergodic theorem on complete random inner product modules. Front. Math. China 6(5), 965-985 (2011)

13. Muštari, DK: On almost sure convergence in linear spaces of random variables. Teor. Veroâtn. Ee Primen. 15(2) 351-357 (1970)

14. Taylor, RL: Convergence of elements in random normed spaces. Bull. Aust. Math. Soc. 12, 31-47 (1975)

15. Beck, A, Schwartz, JT: A vector-valued random ergodic theorem. Proc. Am. Math. Soc. 8, 1049-1059 (1957)

16. Filipović, D, Kupper, M, Vogelpoth, N: Separation and duality in locally $L^{0}$-convex modules. J. Funct. Anal. 256, 3996-4029 (2009)

17. Dunford, N, Schwartz, JT: Linear Operators (I). Interscience, New York (1957)

18. Schweizer, B, Sklar, A: Probabilistic Metric Spaces. Elsevier, New York (1983). Reissued by Dover Publications, New York (2005)

19. Guo, TX: Module homomorphisms on random normed modules. Northeast. Math. J. 12, 102-114 (1996)

doi:10.1186/1029-242X-2012-150

Cite this article as: Zhang: On conditional mean ergodic semigroups of random linear operators. Journal of Inequalities and Applications 2012 2012:150.

\section{Submit your manuscript to a SpringerOpen ${ }^{\circ}$ journal and benefit from:}

- Convenient online submission

- Rigorous peer review

Immediate publication on acceptance

- Open access: articles freely available online

- High visibility within the field

- Retaining the copyright to your article 